\title{
Diversidad de macroinvertebrados acuáticos en quebradas con diferente cobertura ribereña en el bosque Protector Murocomba, Ecuador
}

\author{
Juan Pablo Urdanigo ${ }^{1}$, Mariela Díaz Ponce ${ }^{1}$, Carolina Tay-Hing Cajas ${ }^{1}$, \\ Carlos Sánchez Fonseca², Ronny Yong Benitez ${ }^{1}$, Katherine Armijo Albán ${ }^{1}$, \\ Norma Guerrero Chúez ${ }^{1} \&$ Néstor Javier Mancera-Rodríguez ${ }^{3 *}$
}

1. Universidad Técnica Estatal de Quevedo. Facultad de Ciencias Ambientales, Carrera de Ingeniería en Gestión Ambiental, Km. 1⁄1⁄2 vía Sto. Domingo de los Tsáchilas, Quevedo, Los Ríos Ecuador; jurdanigo@uteq.edu.ec, mdiaz@uteq.edu.ec, ctayhing@uteq.edu.ec,emilio3.5@hotmail.com,kata_armi@hotmail.com,nguerrero@uteq.edu.ec

2. Universidad Técnica Estatal de Quevedo. Facultad de Ciencias Ambientales, Carrera de Ingeniería Forestal, Km. 1⁄2 vía Sto. Domingo de los Tsáchilas, Quevedo, Los Ríos Ecuador; carlos_sanchez70@hotmail.com

3. Universidad Nacional de Colombia, Sede Medellín. Departamento de Ciencias Forestales, Grupo Ecología y Conservación de Fauna Silvestre, Calle 59A No. 63-20, Bloque 20, oficina 211, Medellín, Colombia; njmancer@unal.edu.co

* Correspondencia

Recibido 15-XI-2018. Corregido 23-IV-2019. Aceptado 11-VII-2019.

\begin{abstract}
Diversity of aquatic macroinvertebrates along creeks with different riparian cover in Murocomba Protector Forest, Ecuador. Land-use change from native forests to agriculture, livestock and exotic forest plantations is a main factor affecting riparian vegetation and aquatic communities. The aim of this study was to assess responses of aquatic macroinvertebrate assemblages to riparian cover change in Murocomba protective forest, Valencia, Ecuador. Aquatic macroinvertebrates were collected and physicochemical variables were measured during dry (June-October 2014) and rainy (December-April 2015) seasons along three creeks with different riparian cover: native forests Q1-CBN, pasture for agricultural and livestock activities Q2-CAG, and exotic forest plantations Q3-CPF. According to the Macroinvertebrate Family Biotic Index (FBI) and the Biological Monitoring Working Party Index (BMWP/Col) the best conditions existed in the creek Q1-CBN, while the Ephemeroptera, Plecoptera and Trichoptera Index (EPT) decreased in the creek Q2-CAG during the rainy season. The Shannon-Weaver index showed higher values in Q1-CBN and Q3-CPF in the dry period, and the dominance increased in the Q3-CPF in rainy season. A total of 6583 macroinvertebrates were collected. Trichoptera was the most abundant order and Hidropsychidae the most abundant family. The Coleoptera order showed greater abundance in Q1-CBN and Diptera in Q2-CAG, while Hemiptera showed the lowest abundance in Q3-CPF. Psephenidae, Chironomidae, Leptophlebiidae, Veliidae, Simuliidae, Baetidae and Leptohyphidae were associated with the highest values of total dissolved solids and $\mathrm{pH}$, as well as higher values of IBF and lower values of EPT, BMWP, and depth of the channel in Q2-CAG, whereas Leptoceridae, Elmidae, Ptilodactylidae and Philopotamidae were associated with higher values of EPT, BMWP and depth of the channel in Q1-CBN and Q3-CPF. A negative influence of anthropic activities on the physicochemical variables and structure of the macroinvertebrate assemblages was evidenced. This indicates that the riparian vegetation is a factor that determines the establishment and structure of aquatic communities.
\end{abstract}

Key words: aquatic insects; biomonitoring; land-uses; conservation; biological indexes.

Urdanigo, J. P., Díaz Ponce, M., Tay-Hing Cajas, C., Sánchez Fonseca, C., Yong Benitez, R., Armijo Albán, K., Guerrero Chúez, N., \& Mancera-Rodríguez, N. J. (2019). Diversidad de macroinvertebrados acuáticos en quebradas con diferente cobertura ribereña en el bosque Protector Murocomba, Ecuador. Revista de Biología Tropical, 67(4), 861-878. 
Los cambios en el uso del suelo de bosques nativos a tierras usadas en agricultura, ganadería y plantaciones forestales, afectan las comunidades acuáticas, ya que la pérdida de vegetación ribereña altera directa e indirectamente la calidad del agua, el tipo de sustrato y afecta las condiciones físicas del hábitat acuático y las comunidades bióticas asociadas (e.g. Giraldo, Chará, Zúñiga, Chará-Serna \& Pedraza, 2014; Galeano-Rendón, MonsalveCortes, \& Mancera-Rodríguez, 2017), disminuyendo el material alóctono que ingresa al cuerpo hídrico y la disponibilidad de sustratos aportados por los bosques adyacentes (e.g. Bücker, Sondermann, Frede, \& Breuer, 2010; Giraldo et al., 2014), y alterando la estructura y la función trófica de las comunidades de insectos acuáticos (e.g., Lorion \& Kennedy, 2009; Ferrú \& Fierro, 2015; Iñiguez-Armijos, Hampel, \& Breuer, 2018).

Para evaluar los impactos antrópicos en los ecosistemas acuáticos, muchos estudios utilizan indicadores biológicos que proporcionan información sobre la condición ecológica y la evaluación de ecosistemas fluviales, en consecuencia los ensamblajes de macroinvertebrados acuáticos son excelentes indicadores de la calidad del agua debido a la variedad de sensibilidad a la contaminación orgánica y tolerancia a diferentes alteraciones de su hábitat (Figueroa, Palma, Ruiz, \& Niell, 2007; Barba, Lanza, Ramos, \& González, 2013).

Diversos estudios han encontrado que los cambios en el uso de la tierra y por consiguiente en las coberturas vegetales de los márgenes de las quebradas, tienen efectos sobre los ensamblajes de macroinvertebrados acuáticos, con variaciones en la composición taxonómica y de grupos funcionales y una disminución de la diversidad en quebradas con coberturas de bosques nativos, con relación a quebradas con coberturas de plantaciones de bosques exóticos, y de pastos para actividades agrícolas y ganaderas (e.g. Lorion \& Kennedy, 2009; Fierro et al., 2017; Iñiguez-Armijos et al., 2018).

En Ecuador se han desarrollado estudios con macroinvertebrados acuáticos como indicadores biológicos que proporcionan información sobre la condición ecológica de diversos cuerpos hídricos (e.g. Giacometti \& Bersosa, 2006; Arroyo \& Encalada, 2009; Terneus, Hernández, \& Racines, 2012; Álvarez-Mieles et al., 2013; Villamarín, Rieradevall, Paul, Barbour, \& Prat, 2013; Damanik-Ambarita et al., 2016; Liñero et al., 2016), sobre la diversidad taxonómica y funcional en relación con los regímenes de caudal y disturbios hidrológicos o como respuesta a factores ambientales y condiciones fisicoquímicas (e.g., Jacobsen \& Encalada, 1998; Carrera \& Gunkel, 2003; Ríos-Touma, Encalada, \& Prat, 2009, 2011), existiendo poca información acerca de los efectos de los cambios de la vegetación riparia sobre las comunidades de macroinvertebrados acuáticos (e.g.; Bojsen \& Jacobsen, 2003; Bücker et al., 2010; Iñiguez-Armijos, Leiva, Frede, Hampel \& Breuer, 2014; Iñiguez-Armijos et al., 2016; Iñiguez-Armijos et al., 2018).

Dado el incremento de pérdida de bosques por cambios en el uso de suelo con el consecuente aumento de afectaciones por actividades antrópicas y la falta de conocimiento de la respuesta del ensamblaje de macroinvertebrados a dichas alteraciones, el presente estudio espera proporcionar información sobre los efectos de las variaciones de la vegetación de ribera asociada a tres quebradas tropicales con diferentes coberturas de vegetación riparia (bosque nativo, pastos/cultivos y plantación forestal) sobre las comunidades de macroinvertebrados en dos períodos hidrológicos (seca vs. lluviosa) contrastantes en el bosque protector Murocomba, Provincia de Los Ríos, Ecuador. Se plantea como hipótesis de investigación que la quebrada con márgenes riparios con uso de bosque nativo presentará mejor condición ecológica, por lo tanto, la diversidad taxonómica (estructura y composición) a nivel de familias será mayor que en zonas con coberturas en pastos para uso agrícola y ganadero y de plantación forestal.

La información de este estudio servirá como base ecológica para futuros estudios de calidad de agua mediante el empleo de macroinvertebrados acuáticos como índices biológicos, contribuirá a la generación de 
información sobre las respuestas del ensamblaje de macroinvertebrados acuáticos al cambio en la cobertura ribereña de quebradas, útil para la formulación de estrategias efectivas de conservación, restauración y manejo de bosques de ribera en el área de bosque protector Murocomba y a la mitigación de los efectos negativos de las actividades agropecuarias sobre las zonas de margen de quebradas y cuerpos hídricos.

\section{MATERIALES Y MÉTODOS}

Área de estudio: El trabajo se realizó en tres quebradas de la zona del Bosque Protector Murocomba, en la provincia Los Ríos, Ecuador, con diferentes tipos de cobertura vegetal en sus márgenes. El rango altitudinal de los sitios de estudio se encuentra entre los 415 y 613 m, y de acuerdo a Holdridge (1982) corresponde a una zona de bosque húmedo premontano (bh-PM), con una temperatura que oscila entre 18 y $23{ }^{\circ} \mathrm{C}$.

La quebrada La Victoria (Q1-CBN) (0³8'18.1" S \& 7908'53.7" W, 613 m.s.n.m.) presenta cobertura de bosque nativo secundario en sus dos márgenes en buen estado de conservación; tiene una amplia franja de vegetación ribereña y un dosel que alcanza más de $15 \mathrm{~m}$ de altura, con presencia de vegetación arbórea nativa y especies como Guachapelí (Albizia guachapeli Kunth), Naranjillo (Trichanthera gigantea Humb. \& Bonpl.), Tillo (Brosimum acutifolium Huber), Clavellín (Brownea herthae Harms), Caucho (Ficus elastica Hornem), Guarumo (Cecropia peltata L.), Cedrillo (Terminalia sp.), Laurel blanco (Cordia alliodona Johnst.), Guabo (Inga edulis Mart.), Pambil (Iriartea sp.), Tomate de árbol de montaña (Solanum betaceum Cav.), Moral Fino (Chlorophora tinctoria L. Gaudich), Balsa (Ochroma pyramidale Cav. ex Lam. Urb.), entre otras. La quebrada El Congo (Q2-CAG) (0³7'32.9'" S - 7909' 07.7'W, 533 m.s.n.m.) corresponde a la zona de cobertura en pastos con actividades agrícolas y ganaderas; sus márgenes están cubiertos principalmente por pastos y algunos cultivos de maíz, naranjilla, plátano y cacao. La quebrada La Damita (Q3-CPF) (0³8'38.2” S
\& 79010'46.7' W, 415 m.s.n.m.) corresponde a la zona de cobertura de plantaciones forestales de especies no nativas de la zona como Melina (Gmelina arborea Roxb.) y Pachaco (Schizolobium parahybum Blake) de más de 5 ha de extensión con árboles de cerca de $7 \mathrm{~m}$ de altura.

\section{Variables fisicoquímicas e hidrológicas:}

Se midieron las siguientes variables in situ: oxígeno disuelto $(\mathrm{mg} / \mathrm{L}), \mathrm{pH}$, conductividad $(\mu \mathrm{S} / \mathrm{cm})$, temperatura superficial del agua $\left({ }^{\circ} \mathrm{C}\right)$, y sólidos totales disueltos STD $(\mathrm{mg} / \mathrm{L})$ con un medidor Hanna HI-9829. El ancho y la profundidad fueron medidos con flexómetro, y una vara graduada en $\mathrm{cm}$. El caudal (Q) se determinó a través del método del objeto flotante, siendo $\mathrm{Q}=(\mathrm{Fc}) \times(\mathrm{A}) \times(\mathrm{V})$, donde Fc representa el factor de corrección (0.70) para canal en tierra con profundidad $>15 \mathrm{~cm}$, A representa el área y V la velocidad. La velocidad de la corriente se midió con la ayuda de un objeto flotador en cuatro puntos del cauce, midiendo el tiempo que demoró en recorrer cinco metros de distancia. El área de la sección transversal se calculó midiendo el ancho del río en cuatro puntos distintos de las márgenes y sobre esta referencia se midió la profundidad en puntos consecutivos ubicados cada $20 \mathrm{~cm}$.

Recolecta e identificación de macroinvertebrados acuáticos: $L a$ recolección de macroinvertebrados acuáticos se realizó en dos períodos hidrológicamente distintos: (i) temporada seca entre junio y noviembre de 2014 y (ii) temporada lluviosa entre diciembre de 2014 y abril de 2015. En cada período hidrológico se realizaron cuatro muestreos con un intervalo de 45 días entre cada muestreo. Los macroinvertebrados acuáticos se recolectaron usando una red D-net de $405 \mathrm{~cm}^{2}$ y con malla de $500 \mu \mathrm{m}$ ubicada en sentido contrario de la corriente, para lo cual se seleccionaron 20 puntos al azar a lo largo del tramo de $100 \mathrm{~m}$ en cada quebrada, removiendo hojarascas y sedimento in situ en el cauce de las quebradas. El material recolectado fue etiquetado y almacenado en recipientes con alcohol al $70 \%$. La identificación taxonómica de los especímenes se realizó hasta 
el nivel de familia utilizando un estereoscopio y claves taxonómicas especializadas (Roldán, 2003; Domínguez \& Fernández, 2009) y las muestras se encuentran depositadas en el laboratorio de Microbiología y Entomología de la Universidad Técnica Estatal de Quevedo.

Índices biológicos: Para determinar la condición ecológica se calcularon los valores del Índice Biótico de Familias (IBF), el Índice de porcentaje de individuos de Ephemeroptera, Plecoptera y Trichoptera (EPT) y el Índice Biological Monitoring Working Party (BMWP/Col), para cada estación de muestreo y período climático.

Análisis de datos: Para establecer si existieron diferencias significativas en los valores de las variables fisicoquímicas y los valores de caudal, así como de los índices IBF, EPT y BMWP/Col entre períodos hidrológicos y entre quebradas con diferente tipo de cobertura, se aplicaron análisis de varianza unidireccionales (ANOVA) a cada variable, previa validación de los supuestos de normalidad (KolmogorovSmirnov) y homocedasticidad (prueba de Levene) y se realizaron comparaciones evaluadas mediante la prueba de Tukey. Cuando los datos no cumplieron con los supuestos de normalidad y homocedasticidad se utilizó la prueba no paramétrica de Kruskall-Wallis.

Se determinó el número de familias e individuos, y se calcularon los índices de ShannonWeaver, Dominancia, Equidad de Simpson, número de taxones raros (abundancia inferior al $3 \%$ ), para cada estación de muestreo y período hidrológico, utilizando el programa estadístico PAST (Hammer, Harper, \& Ryan, 2001). Los valores de los índices de diversidad Shannon-Weaver y dominancia de Simpson entre estaciones y períodos se compararon mediante pruebas de $t$ de Hutcheson (Zar, 1999).

Se definieron los patrones de variación en la composición de familias del ensamblaje de macroinvertebrados entre quebradas con diferentes coberturas con respecto a las variables ambientales y a los valores de los Índices IBF, EPT y BMWP-Col, mediante análisis discriminante canónico (ADC), que se realizó sobre datos de abundancia de los taxones que presentaron una frecuencia mayor al $5 \%$ y una abundancia superior a $3 \%$ (Gauch, 1982; RíosPulgarín, Barletta, Arango-Jaramillo, \& Mancera-Rodríguez, 2016a). En este análisis, los taxones que ayudan a explicar las diferencias se representan como vectores cuya longitud y dirección es proporcional a su contribución para explicar la variabilidad (Ríos-Pulgarín, Barletta, \& Mancera-Rodríguez, 2016b). Todos los análisis se desarrollaron en versiones $\mathrm{R}$ Software 3.1.1 (R Core Team, 2012) y RWizard (Guisande, 2015).

\section{RESULTADOS}

Variables fisicoquímicas e hidrológicas: La quebrada La Victoria (Q1-CBN) presentó un sustrato pedregoso y rocoso, con pendiente inclinada y con caudal medio de $0.292 \mathrm{~m}^{3} / \mathrm{s}$ en período seco y de $0.590 \mathrm{~m}^{3} / \mathrm{s}$ en período de lluvias. Presentó un ancho medio de $4.25 \mathrm{~m}$ en el período seco y de $4.50 \mathrm{~m}$ en el período de lluvias y una profundidad entre 25 y $30 \mathrm{~cm}$. La quebrada El Congo (Q2- CAG), presentó un sustrato pedregoso, con pendiente plana y con caudal medio de $0.108 \mathrm{~m}^{3} / \mathrm{s}$ en período seco y de $0.815 \mathrm{~m}^{3} / \mathrm{s}$ en período de lluvias. Presentó un ancho medio de $3.85 \mathrm{~m}$ en el período seco y de $5.70 \mathrm{~m}$ en el período de lluvias y una profundidad entre 14 y $24 \mathrm{~cm}$. Por último, la quebrada La Damita (Q3-CPF) posee un sustrato arenoso con pendiente plana y con caudal medio de $0.365 \mathrm{~m}^{3} / \mathrm{s}$ en período seco y de $0.829 \mathrm{~m}^{3} / \mathrm{s}$ en período de lluvias. Presentó un ancho medio de $5.70 \mathrm{~m}$ en el período seco y de $6.30 \mathrm{~m}$ en el período de lluvias y una profundidad media entre 15 y $22 \mathrm{~cm}$ (Tabla 1).

Se encontraron diferencias significativas en los valores de oxígeno disuelto entre períodos hidrológicos para las tres quebradas estudiadas (Kruskal-Wallis, $\mathrm{P}<0.05$ ) con mayores valores medios durante el período de lluvia entre 9.4 y $9.7 \mathrm{mg} / \mathrm{l}$ y una marcada disminución en el período seco con valores medios entre 4.8 y $5.4 \mathrm{mg} / \mathrm{l}$ (Tabla 1). Se encontró también diferencia significativa en la quebrada El 
Congo (Q2-CAG) que tiene márgenes con uso de suelo agrícola-ganadero para los valores de caudal (Kruskal-Wallis, $\mathrm{P}=0.043$ ) que fueron mayores en el período de lluvia. Las demás variables fisicoquímicas no presentaron diferencias significativas entre períodos hidrológicos para cada quebrada, aunque la temperatura superficial del agua fue mayor para las tres quebradas en la temporada seca (Tabla 1).

Variabilidad taxonómica de macroinvertebrados acuáticos entre quebradas: En los cuerpos hídricos evaluados se recolectaron un total de 6583 macroinvertebrados acuáticos pertenecientes a dos filos, tres clases, 13 órdenes y 54 familias. En el período seco se recolectaron 2914 individuos de 52 familias y en la época lluviosa 3669 de 34 familias (Tabla2). De manera general el orden Trichoptera con
2590 individuos (39.3\%) fue el mejor representado, seguido de los órdenes Coleoptera con 1547 individuos (23.5\%) y Ephemeroptera con 995 individuos (15.1\%). A nivel de familias la más abundante fue Hydropsychidae con 2001 individuos (30.40\%), seguida de Elmidae con 1079 individuos (16.4\%).

A nivel de órdenes, se presentaron diferencias en sus abundancias entre las tres quebradas con diferente cobertura de vegetación en sus márgenes. El orden más abundante en las tres fue Trichoptera y aunque la diferencia no fue significativa, la quebrada con cobertura de plantación forestal (Q3-CPF) presentó un valor mayor con un $46.6 \%$ de abundancia, seguida de la quebrada con cobertura de bosque nativo (Q1-CBN) con $38.0 \%$ y la quebrada con cobertura agrícola y ganadera (Q2-CAG) que presentó el menor valor con $27.5 \%$ (Fig. 1A).

TABLA 1

Valores de los índices y características químicas durante las temporadas seca y lluviosa en tres quebradas con diferentes coberturas riparias en el Bosque Protector Murocomba, Ecuador

TABLE 1

Values of the indexes and chemical characteristics during dry and rainy seasons in three creeks with different riparian cover in the Murocomba Protector Forest, Ecuador

\begin{tabular}{lcccccc} 
& \multicolumn{2}{c}{ Q1-CBN } & \multicolumn{2}{c}{ Q2-CAG } & \multicolumn{2}{c}{ Q3-CPF } \\
& Seca & Lluvia & Seca & Lluvia & Seca & Lluvia \\
Número de familias & 36 & 26 & 36 & 25 & 39 & 25 \\
Número de individuos & 885 & 1192 & 768 & 797 & 1261 & 1680 \\
Índice de Shannon $\left(\mathrm{H}^{\prime}\right)$ & 2.56 & 2.30 & 2.57 & 2.59 & 2.52 & 1.59 \\
Dominancia $(\mathrm{D})$ & 0.12 & 0.14 & 0.12 & 0.11 & 0.12 & 0.36 \\
Equidad de Simpson $(1-\mathrm{D})$ & 0.88 & 0.86 & 0.88 & 0.89 & 0.88 & 0.64 \\
Número de taxones raros & 29 & 19 & 27 & 16 & 31 & 19 \\
Índice IBF & $4.3 \pm 0.2$ & $3.9 \pm 0.7$ & $4.9 \pm 0.7$ & $5.3 \pm 0.9$ & $5.0 \pm 0.0$ & $5.1 \pm 0.2$ \\
Taxones EPT & $7.3 \pm 3.0$ & $7.5 \pm 0.6$ & $7.0 \pm 1.4$ & $6.8 \pm 1.3$ & $7.5 \pm 2.9$ & $7.0 \pm 0.8$ \\
Índice \% EPT & $51.4 \pm 19.6$ & $61.7 \pm 9.3$ & $60.5 \pm 2.7$ & $51.4 \pm 10.2$ & $47.8 \pm 19.6$ & $71.9 \pm 9.4$ \\
BMWP/Col & $111.3 \pm 4.3$ & $120.5 \pm 36.8$ & $105.5 \pm 6.6$ & $106.5 \pm 25.2$ & $98.0 \pm 5.5$ & $115.8 \pm 17.7$ \\
O (mg/L) & $\mathbf{4 . 9} \pm 0.6$ & $\mathbf{9 . 7} \pm 1.3$ & $\mathbf{5 . 4} \pm 0.1$ & $\mathbf{9 . 5} \pm 1.3$ & $\mathbf{4 . 8} \pm 0.5$ & $\mathbf{9 . 4} \pm 1.1$ \\
pH & $7.2 \pm 0.8$ & $7.2 \pm 0.1$ & $7.4 \pm 1.0$ & $7.3 \pm 0.1$ & $7.3 \pm 1.0$ & $7.2 \pm 0.1$ \\
Conductividad $(\mu \mathrm{S} \mathrm{cm}-1)$ & $184.0 \pm 60.8$ & $197.3 \pm 87.3$ & $203.0 \pm 84.2$ & $196.8 \pm 55.1$ & $174.6 \pm 28.6$ & $187.5 \pm 50.0$ \\
Temperatura agua $\left({ }^{\circ} \mathrm{C}\right)$ & $21.8 \pm 3.1$ & $16.4 \pm 6.7$ & $23.5 \pm 3.0$ & $17.2 \pm 7.0$ & $22.8 \pm 1.26$ & $17.6 \pm 6.0$ \\
sólidos totales disueltos $(\mathrm{mg} / \mathrm{L})$ & $85.8 \pm 29.2$ & $83.8 \pm 46.0$ & $97.0 \pm 36.0$ & $90.5 \pm 27.7$ & $90.3 \pm 13.5$ & $86.8 \pm 25.8$ \\
Ancho medio $(\mathrm{cm})$ & $425 \pm 10$ & $450 \pm 34.6$ & $385 \pm 64$ & $570 \pm 175.1$ & $570 \pm 154.5$ & $630 \pm 34.6$ \\
Profundidad media $(\mathrm{cm})$ & $25.0 \pm 5.4$ & $29.2 \pm 5.9$ & $14.4 \pm 2$ & $23.8 \pm 4.3$ & $15.7 \pm 4.2$ & $21.2 \pm 9.6$ \\
Caudal medio $\left(\mathrm{m}^{3} / \mathrm{s}\right)$ & $0.292 \pm 0.17$ & $0.590 \pm 0.38$ & $\mathbf{0 . 1 0 8} \pm 0.16$ & $\mathbf{0 . 8 1 5} \pm 0.43$ & $0.365 \pm 0.28$ & $0.830 \pm 0.77$ \\
\hline
\end{tabular}

Q1-CBN, bosque nativo; Q2-CAG, pastos para actividades agrícolas y ganaderas; Q3-CPF, plantación forestal. $\mathrm{Q} 1-\mathrm{CBN}$, native forest, Q2-CAG, pasture for agricultural and livestock activities; Q3-CPF, forest plantation. 
TABLA 2

Número total de macroinvertebrados capturados durante las temporadas seca y lluviosa en tres quebradas con diferentes coberturas riparias en el Bosque Protector de Murocomba, Ecuador

TABLE 2

Totals number of macroinvertebrates caught during dry and rainy seasons in three creeks with different riparian cover in the Murocomba Protector Forest, Ecuador

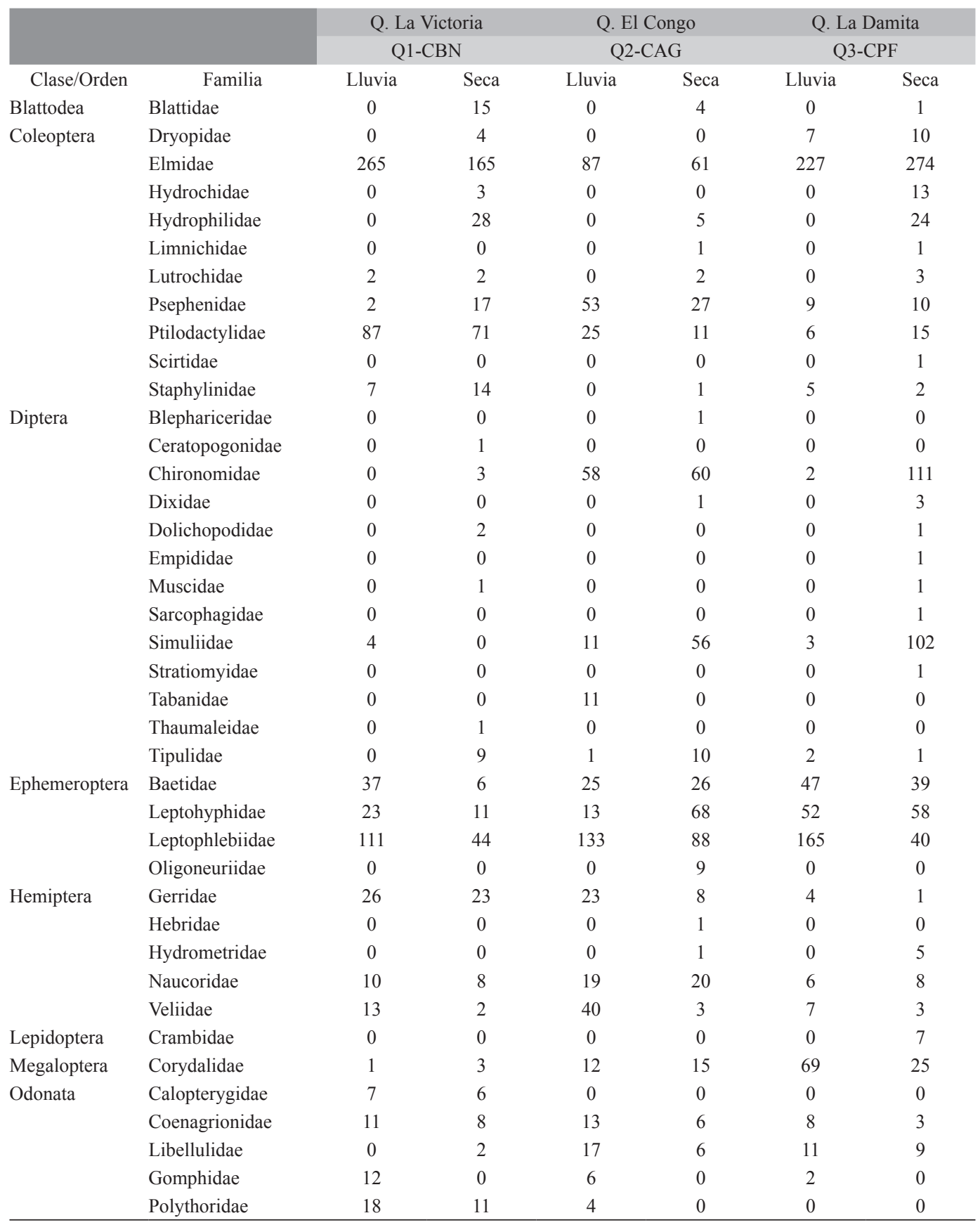


TABLA 2 (Continuación) / TABLE 2 (Continued)

\begin{tabular}{llcccccc} 
& & \multicolumn{2}{c}{ Q. La Victoria } & \multicolumn{2}{c}{ Q. El Congo } & \multicolumn{2}{c}{ Q. La Damita } \\
& & \multicolumn{2}{c}{ Q1-CBN } & \multicolumn{2}{c}{ Q2-CAG } & \multicolumn{2}{c}{ Q3-CPF } \\
Plecoptera & Perlidae & 81 & 99 & 46 & 43 & 8 & 153 \\
Trichoptera & Calamoceratidae & 3 & 13 & 0 & 1 & 0 & 0 \\
& Glossosomatidae & 0 & 1 & 0 & 3 & 0 & 0 \\
& Helicopsychidae & 5 & 6 & 10 & 6 & 0 & 0 \\
& Hydrobiosidae & 13 & 2 & 0 & 6 & 0 & 23 \\
& Hydropsychidae & 229 & 167 & 175 & 212 & 965 & 253 \\
& Hydroptilidae & 0 & 0 & 2 & 0 & 0 & 3 \\
& Leptoceridae & 217 & 129 & 6 & 1 & 6 & 24 \\
Osopoda & Odontoceridae & 0 & 0 & 6 & 2 & 2 & 1 \\
Gastropoda & Philopotamidae & 2 & 2 & 0 & 0 & 62 & 29 \\
& Polycentropodidae & 0 & 0 & 0 & 1 & 1 & 1 \\
& Pseudothelphusidae & 3 & 2 & 1 & 1 & 4 & 0 \\
\hline
\end{tabular}

Q1-CBN, bosque nativo; Q2-CAG, pastos para actividades agrícolas y ganaderas; Q3-CPF, plantación forestal

$\mathrm{Q} 1-\mathrm{CBN}$, native forest, Q2-CAG, pasture for agricultural and livestock activities; Q3-CPF, forest plantation

El orden Coleoptera presentó mayor abundancia en la quebrada Q1-CBN con un 32.1\% y fue significativamente diferente a la quebrada Q2-CAG con un $17.4 \%(\mathrm{P}=0.017)$ y no presento diferencia significativa con la quebrada Q3-CPF, mientras que el orden Diptera fue más abundante en la quebrada con cobertura agrícola y ganadera (Q2-CAG) con un 13.4\% y significativamente diferente de Q1-CBN que presentó solamente un $1.0 \%(\mathrm{P}=0.022)$. El orden Hemiptera presentó la menor abundancia en la quebrada Q3-CPF y fue significativamente diferente de $\mathrm{Q} 1-\mathrm{CBN}(\mathrm{P}=0.036)$ y de Q2-CAG (P = 0.041), y el orden Megaloptera presentó la menor abundancia en la quebrada Q1-CBN y fue significativamente diferente de Q2-CAG $(\mathrm{P}=0.003)$ y de Q3-CPF $(\mathrm{P}=0.004)$ (Fig. 1A).

Las familias Hydropsychidae, Elmidae, Leptophlebiidae y Perlidae representaron el $62.1 \%$ de la abundancia relativa para el total de individuos colectados en las tres quebradas. El análisis canónico discriminante basado en la abundancia de familias de macroinvertebrados y las variables ambientales separó la quebrada Q2-CAG con uso de suelo agrícola y ganadero y cobertura de pastos en sus márgenes, de las quebradas Q1-CBN con uso de bosque nativo y la quebrada Q3-CPF de plantación forestal y representó el $91.7 \%$ de la varianza en el primer eje canónico. Para la quebrada Q2-CAG, las familias que contribuyeron a la variación fueron principalmente Psephenidae, Chironomidae, Leptophlebiidae, Veliidae, Simuliidae, Baetidae y Leptohyphidae, que se asociaron con los mayores valores de sólidos disueltos totales y $\mathrm{pH}$, así como a mayores valores de IBF y menores valores de los índices EPT y BMWP y de profundidad del cauce (Fig. 2). En las quebradas Q1-CBN y Q3-CPF las familias que más contribuyeron a la variación fueron Leptoceridae, Elmidae, Ptilodactylidae y Philopotamidae, esta última, principalmente en Q3-CPF y se asociaron con mayores valores de los índices EPT y BMWP y con mayor profundidad del cauce (Fig. 2).

Variabilidad taxonómica de macroinvertebrados acuáticos entre períodos Hidrológicos: En la Quebrada La Victoria (Q1-CBN) se identificaron 38 familias y se recolectaron un total de 2077 individuos de macroinvertebrados, de los cuales 885 individuos de 36 familias fueron recolectados en el período seco 

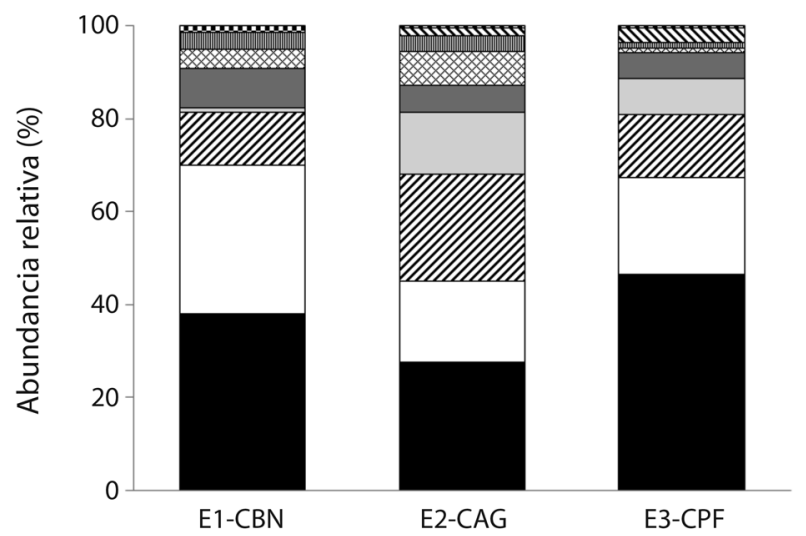

Otros

$\mathbb{N}$ Megaloptera

Od Odonata

凶 Hemiptera

$\square$ Plecoptera

$\square$ Diptera

$\square$ Ephemeroptera

$\square$ Coleoptera

- Trichoptera

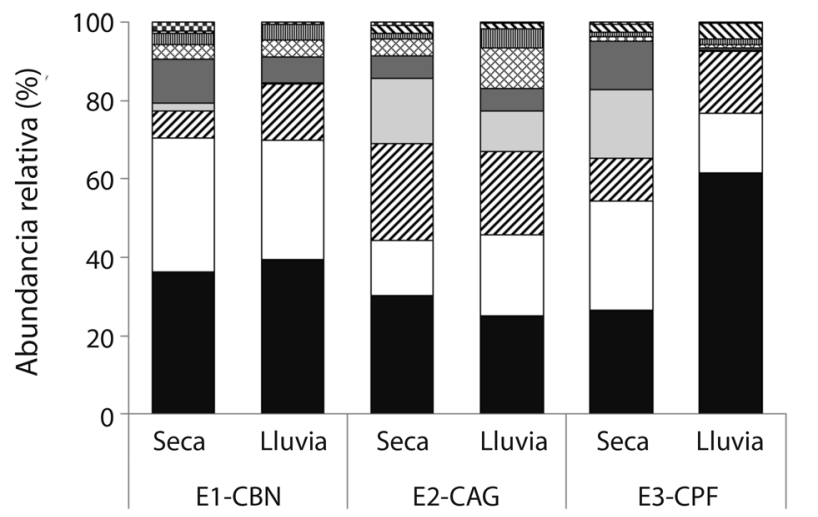

O Otros

$\mathbb{\Delta}$ Megaloptera

Odonata

ه Hemiptera

$\square$ Plecoptera

$\square$ Diptera

Ephemeroptera

$\square$ Coleoptera

- Trichoptera

Tipo de estación

Fig. 1. Abundancia relativa de órdenes de macroinvertebrados (A) para cada período hidrológico (B) en tres quebradas con diferentes coberturas riparias (Q1-CBN, bosque nativo; Q2-CAG, pastos para actividades agrícolas y ganaderas; Q3-CPF, plantación forestal) en el Bosque Protector de Murocomba, Valencia, Ecuador.

Fig. 1. Relative abundance of macroinvertebrate orders (A), for hydrological periods (B) in three creeks with different riparian cover (Q1-CBN, native forest, Q2-CAG, pasture for agricultural and livestock activities; E3-CPF, forest plantation) in the Murocomba Protector Forest, Valencia, Ecuador.

y 1192 individuos de 26 familias en período de lluvias (Tabla 1). Los órdenes más abundantes tanto en época seca como en lluvias fueron Trichoptera (36.2 y $39.3 \%$, respectivamente) y Coleoptera (34.4 y $30.5 \%$, respectivamente). Se observó un aumento significativo del orden Ephemeroptera en el período de lluvia con un $14.3 \%$, con relación a la época seca con un $6.9 \%(\mathrm{P}=0.031)$ (Fig. 1B). En época seca las familias más abundantes fueron Hydropsychidae con un $18.9 \%$, Elmidae con $18.6 \%$ y Leptoceridae con $14.6 \%$, y en época lluviosa la familia Elmidae fue la más abundante con un $22.2 \%$, seguida por Hydropsychidae con $19.2 \%$ y Leptoceridae con $18.2 \%$ de individuos (Tabla 2).

Para la Quebrada El Congo de cobertura en pastos y uso agrícola y ganadero (Q2-CAG) se recolectaron 1565 individuos pertenecientes a 40 familias, de los cuales 768 individuos de 36 familias fueron recolectados en el período seco y 797 individuos de 25 familias en período de lluvias (Tabla 1). El orden más abundante tanto en época seca como en lluvias fue Trichoptera (30.2 y $25.0 \%$, respectivamente). Se observó una mayor abundancia del orden 


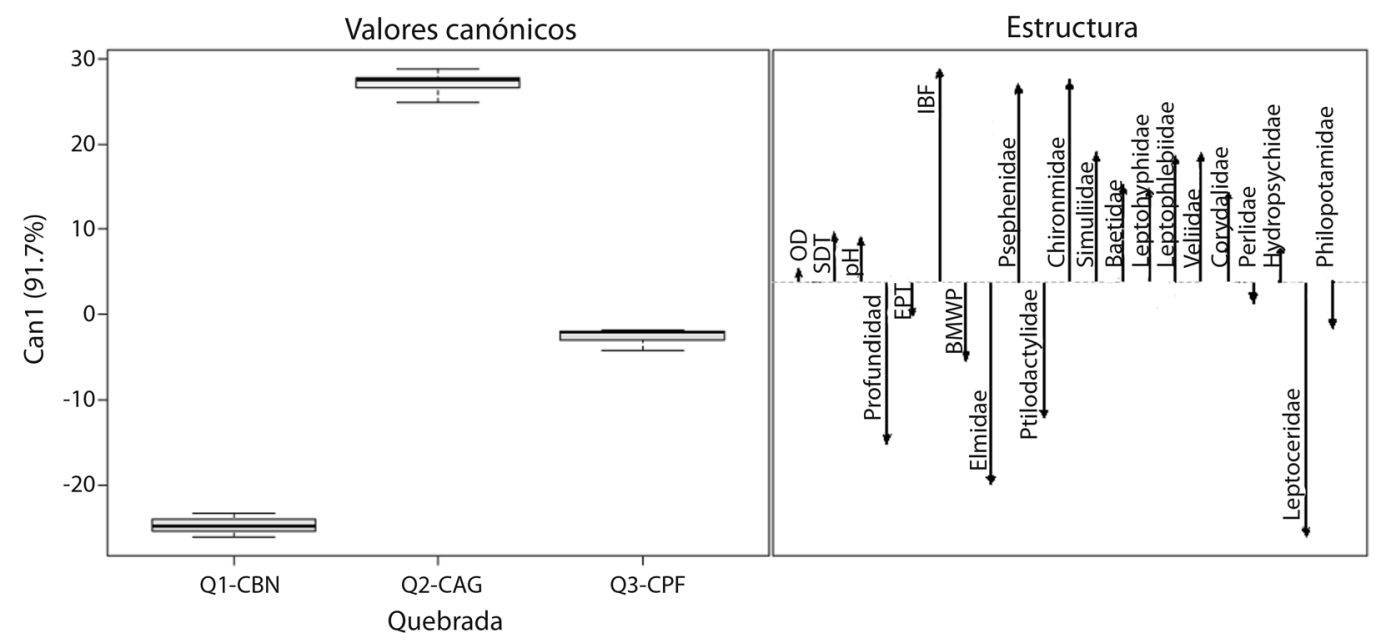

Fig. 2. Análisis discriminante canónico basado en la abundancia de familias de macroinvertebrados y variables ambientales en tres quebradas con diferentes coberturas riparias (Q1-CBN, bosque nativo; Q2-CAG, pastos para actividades agrícolas y ganaderas; Q3-CPF, plantación forestal) en el Bosque Protector de Murocomba, Valencia, Ecuador. OD, oxígeno disuelto; S.D.T., solidos disueltos totales; EPT, Índice Ephemeroptera, Plecoptera y Trichoptera; IBF, Índice biótico de familias; BWMP, Índice Biological Monitoring Working Party.

Fig. 2. Discriminant canonical analysis based on macroinvertebrates families abundance and environmental variables in three creeks with different riparian cover (Q1-CBN, native forest, Q2-CAG, pasture for agricultural and livestock activities; Q3-CPF, forest plantation) in the Murocomba Protector Forest, Valencia, Ecuador. OD, dissolved oxygen; S.D.T., total dissolved solids; EPT, Ephemeroptera, Plecoptera and Trichoptera Index; IBF, Biotic Family Index; BWMP, Biological Monitoring Working Party Index.

Ephemeroptera y una menor abundancia de Coleoptera en los dos períodos, con relación a las quebradas Q1-CBN y Q2-CAG. La abundancia del orden Hemiptera que fue significativamente mayor en el período de lluvias (Fig. 1B). En época seca las familias más abundantes fueron Hydropsychidae con un $27.6 \%$, Leptophlebiidae con $11.5 \%$ y Leptohyphidae con 8.9 $\%$, y en época lluviosa la familia Hydropsychidae fue la más abundante con un 22.0 seguida por Leptophlebiidae con 16.7 \% y Elmidae con $10.9 \%$ de los individuos (Tabla 2).

Para la quebrada La Damita (Q3-CPF) se recolectaron 2941 individuos pertenecientes a 41 familias de macroinvertebrados, de los cuales 1261 individuos de 39 familias fueron recolectados en el período seco y 1680 individuos de 25 familias en período de lluvias (Tabla 1). Se destacó el incremento de Trichoptera en la época de lluvia con un $61.7 \%$ de los individuos, presentando diferencia significativa respecto a la época seca en la misma quebrada ( $\mathrm{P}$
$=0.031) \mathrm{y}$ con los dos períodos hidrológicos de las quebradas Q1-CBN y Q2-CAG (Fig. 1B). Igualmente, se presentó un incremento significativo en el período seco de los órdenes Diptera $(\mathrm{P}=0.031)$ y Plecoptera $(\mathrm{P}=0.030)($ Fig. $1 \mathrm{~B})$. En época seca las familias más abundantes fueron Hydropsychidae con un $20.1 \%$, Elmidae con $21.7 \%$ y Perlidae con $12.1 \%$, y en época lluviosa la familia Hydropsychidae fue la más abundante con un $57.4 \%$ seguida por Elmidae con $13.5 \%$ de los individuos (Tabla 2).

El análisis canónico discriminante (ACD) basado en la abundancia de familias de macroinvertebrados y las variables ambientales separó para los dos períodos evaluados (temporada seca y lluviosa) a la quebrada con uso de suelo agrícola, ganadero y pastos en sus márgenes (Q2-CAG), de las quebradas con uso de bosque nativo (Q1-CBN) y de plantación forestal (Q3-CPF). En época seca el ACD representó el $100.0 \%$ de la varianza en el primer eje canónico y para la quebrada Q2-CAG, 

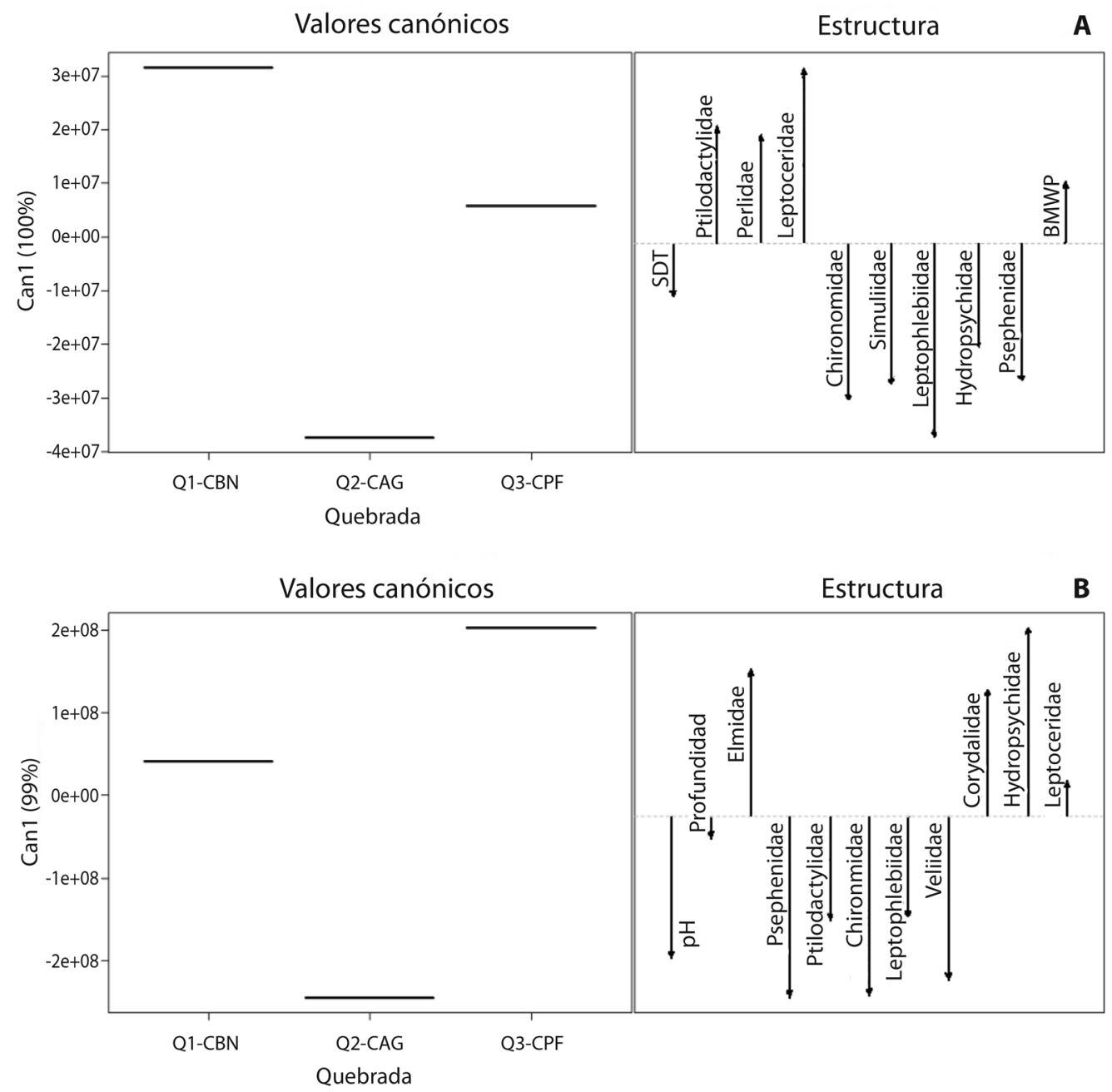

Fig. 3. Análisis discriminante canónico basado en la abundancia de familias de macroinvertebrados y variables ambientales en el período seco (A) y el período de lluvias (B), en tres quebradas con diferentes coberturas riparias (Q1-CBN, bosque nativo; Q2-CAG, pastos para actividades agrícolas y ganaderas; Q3-CPF, plantación forestal) en el Bosque Protector de Murocomba, Valencia, Ecuador. S.D.T., Solidos Disueltos Totales; BWMP, Índice Biological Monitoring Working Party.

Fig. 3. Discriminant canonical analysis based on macroinvertebrates families abundance and environmental variables in dry season (A) and rainy season (B), in three creeks with different riparian cover (Q1-CBN, native forest, Q2-CAG, pasture for agricultural and livestock activities; Q3-CPF, forest plantation) in the Murocomba Protector Forest, Valencia, Ecuador. S.D.T., Total Dissolved Solids; BWMP, Biological Monitoring Working Party Index.

las familias que contribuyeron a la variación fueron Leptophlebiidae, Chironomidae, Simuliidae, Psephenidae, e Hydropsychidae, que se asociaron con los mayores valores de solidos disueltos totales (SDT), así como a menores valores del índice BMWP (Fig. 3A). En las quebradas Q1-CBN y Q3-CPF las familias que más contribuyeron durante la época seca a la variación fueron Leptoceridae, Ptilodactylidae y Perlidae, y se asociaron con mayores valores del índice BMWP (Fig. 3A). En época de lluvia el ACD representó el $99.0 \%$ de la varianza en el primer eje canónico, separando la quebrada Q2-CAG para la que las familias que contribuyeron a la variación fueron principalmente Psephenidae, Chironomidae, 
Veliidae, Ptilodactylidae y Leptophlebiidae, que se asociaron con los mayores valores de $\mathrm{pH}$ y menores valores de profundidad (Fig. 3B). En las quebradas Q1-CBN y Q3-CPF las familias que más contribuyeron durante la época de lluvia a la variación fueron Hydropsychidae y Corydalidae, principalmente en la quebrada con cobertura de plantación forestal Q3-CPF y Elmidae y Leptoceridae en la quebrada Q1-CBN (Fig. 3B).

Índices biológicos: El índice IBF presentó una calidad de agua muy buena para la quebrada con márgenes con cobertura de bosque nativo (Q1-CBN) y calidad regular a buena para las quebradas con cobertura de pastos (Q2-CAG) y con plantaciones forestales (Q3-CPF) (Fig. 4). Los índices EPT y BMWP presentaron calidad buena para las tres quebradas, aunque la quebrada Q3-CPF presentó mayores valores del Índice EPT y la quebrada Q1-CBN del Índice BMWP (Fig. 4). Por períodos hidrológicos, aunque no se encontró diferencia significativa en el índice EPT, se presentó en el período lluvioso mayor abundancia de estos taxones en las quebradas Q1-CBN y Q3-CPF, mientras que para la quebrada Q2-CAG la abundancia de estos fue menor en dicho período (Tabla 1). Los índices IBF y BMWP tampoco presentaron diferencias significativas entre períodos hidrológicos para cada quebrada.

El índice de Shannon-Weaver fue mayor para la quebrada Q2-CAG con valores entre 2.57 en época seca y 2.59 en época lluvia, sin diferencias significativas entre períodos hidrológicos. Por su parte, las quebradas Q1-CBN y Q3-CPF presentaron mayores valores del índice de Shannon-Weaver en el período seco con relación al período de lluvias $(\mathrm{P}<0.001)$, al igual que mayores valores de dominancia ( $\mathrm{P}$ $<0.001$ ), en especial en la quebrada Q3-CPF donde el Índice de diversidad disminuyó de 2.52 en seca a 1.59 en lluvia y la dominancia aumentó de 0.12 en seca a 0.36 en lluvia (Tabla 1). Hubo diferencias significativas en el índice de Shannon-Weaver entre las épocas, las coberturas y la interacción entre épocas y coberturas $(\mathrm{F}=11.87, \mathrm{P}=0.003 ; \mathrm{F}=7.50, \mathrm{P}=0.004 \mathrm{y}$
$\mathrm{F}=8.60, \mathrm{P}=0.002$ ), respectivamente. Los valores de número de familias y número de taxones raros fueron mayores para las tres quebradas en el período seco (Tabla 1).

\section{DISCUSIÓN}

Se determinó una mejor calidad del agua en la quebrada con cobertura de bosque nativo Q1-CBN, con mayores valores de los índices EPT y BMWP, mientras que los valores más bajos se encontraron en la quebrada con márgenes con uso de suelo agrícola y ganadero de cobertura en pastos Q2-CAG. Esto es similar a lo encontrado por Meza, Rubio, Días, \& Walteros (2012) en quebradas de la subcuenca alta del río Chinchiná, en la que la estación con cobertura de pastos presentó menores valores de los índices BMWP y EPT, que las ubicadas en márgenes con cobertura de bosque nativo $\mathrm{y}$ de plantaciones forestales, y también con lo reportado por Guerrero-Chuez et al. (2016) quienes determinaron un índice BMWP con aguas de calidad excelente en el uso de suelo de bosque natural a diferencia de los usos de suelo agrícola y de pastos con aguas de calidad regular, en la microcuenca El Sapanal en Cotopaxi, Ecuador. Al respecto, Iñiguez-Armijos et al. (2014) encontraron que la mayoría de las métricas de macroinvertebrados indican una buena calidad del agua cuando el porcentaje de cobertura de vegetación nativa, tanto en la cuenca como en la zona ribereña inmediata, es superior al $70 \%$, y Galeano-Rendón y Mancera-Rodríguez (2018) encontraron que quebradas con márgenes cubiertos por vegetación nativa presentaron mayores valores de índice BMWP y también mayor promedio de taxones EPT, debido a que dichos ordenes son sensibles a las perturbaciones antrópicas, por lo que suelen estar más asociados a quebradas con zonas ribereñas protegidas. Las quebradas con cobertura de vegetación nativa en sus márgenes presentan ensamblajes más heterogéneos de macroinvertebrados, debido a la abundancia y variedad de hábitats, con una mayor diversidad de taxones intolerantes al estrés ambiental 

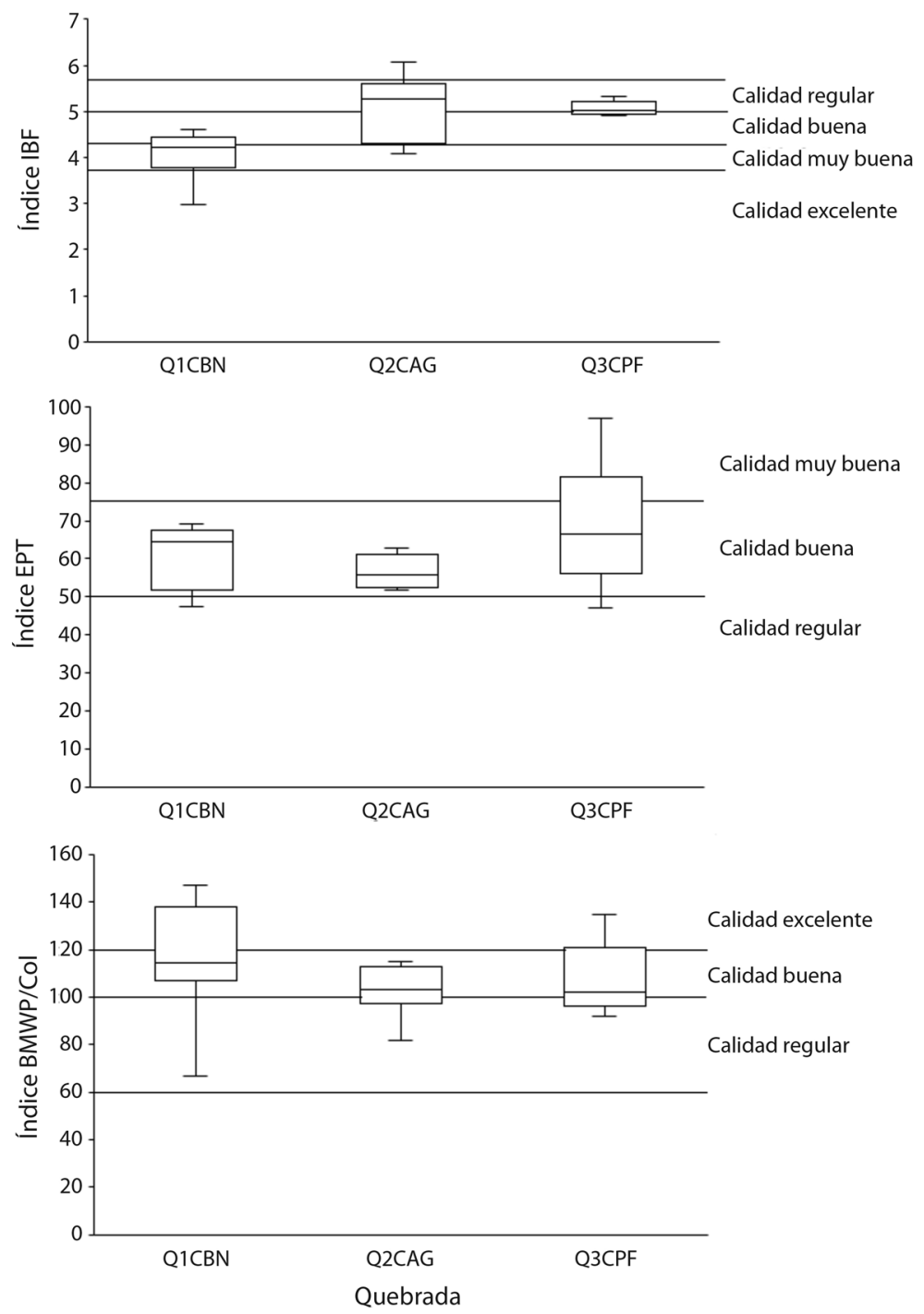

Fig. 4. Puntuación de IBF, EPT y BWMP para tres quebradas con diferentes coberturas riparias (Q1-CBN, bosque nativo; Q2-CAG, pastos para actividades agrícolas y ganaderas; Q3-CPF, plantación forestal) en el Bosque Protector de Murocomba, Valencia, Ecuador. Las líneas indican umbrales de categoría IBF, EPT and BMWP.

Fig. 4. IBF, EPT and BWMP score for three creeks with different riparian cover (Q1-CBN, native forest, Q2-CAG, pasture for agricultural and livestock activities; Q3-CPF, forest plantation) in the Murocomba Protector Forest, Valencia, Ecuador. Lines indicate IBF, EPT and BMWP category thresholds.

(Bojsen \& Jacobsen, 2003; Death \& Collier, 2010; Bücker et al., 2010).

En quebradas con mayor disturbio, se espera una disminución de los taxones sensibles como los EPT y un aumento en la importancia de los taxones tolerantes, reduciendo la diversidad (Bispo \& Oliveira, 2007). En el presente estudio no se observó una disminución marcada en la riqueza y diversidad entre las tres quebradas, pero si una menor abundancia de taxones EPT en la quebrada con márgenes de cobertura en pastos (Q2-CAG), lo cual se debe a que dichos taxones están formados por un grupo diverso de especies que son sensibles 
a los cambios ambientales, como la contaminación y la fragmentación del hábitat (Rosenberg \& Resh, 1993).

Por su parte, a nivel temporal se observó una diminución del índice de Shannon-Weaver, de la riqueza de familias, así como del número de taxones raros durante el período lluvioso. Durante dicho período, la diversidad disminuyó marcadamente en la quebrada con cobertura de plantaciones forestales Q3-CPF, lo cual se asoció a un incremento del orden Trichoptera, en especial por la dominancia de Hydropsychidae. Igualmente se observó una mayor abundancia de taxones EPT durante el período lluvioso en las quebradas Q1-CBN y Q3-CPF, mientras que para la quebrada Q2-CAG, la abundancia de éstos fue menor. Este incremento de taxones puede estar asociado con la mayor cantidad de refugios y entornos heterogéneos que se presentan en quebradas con márgenes con coberturas de bosque, en las que los aumentos en el caudal pueden ser menos drásticos, y en los que muchos macroinvertebrados pueden protegerse contra el efecto de arrastre de la corriente (Scarsbrook \& Townsend, 1993; Townsend, Dolédec, \& Scarsbrook, 1997) y también a una mejora en la calidad del agua en el período lluvioso, que se evidenció en un mayor valor de los índices EPT y BMWP, debido a que el aumento de caudal permite una mayor reducción de la concentración de materia orgánica. Esto último, coincide con lo reportado por Bispo, Oliveira, Bini, y Sousa (2006) quienes encontraron un pico de abundancia de macroinvertebrados al comienzo de la temporada de lluvias asociada con una mejora de calidad de agua. Estos autores destacan que probablemente, los efectos positivos de la dilución de contaminantes observados se pierden con mayores tasas de precipitación e incremento de caudal, lo cual también puede explicar la disminución en taxones EPT durante el período lluvioso en la quebrada Q2-CAG ya que ésta, fue la única en la que se presentó un aumento significativo del caudal. Bispo et al. (2006) destacan que la precipitación explicó una parte importante de la variación temporal en la abundancia de EPT, especialmente en sitios de mayor caudal con una disminución en la abundancia durante la temporada de lluvias.

Esta disminución en taxones EPT en la quebrada de cobertura agrícola y ganadera (Q2-CAG) coincide con lo reportado por Schmitt, Siegloch, da Silva, Lisboa, y Petrucio (2016) quienes también encontraron una baja abundancia de EPT durante los períodos de alta precipitación probablemente causada por un aumento en el flujo de agua, lo que produce un consecuente efecto de deriva de los individuos, la remoción de insectos y una reducción de su abundancia local (Flecker \& Feifarek, 1994), así como con otros estudios en quebradas Andinas, que también reportan una menor abundancia del ensamblaje de macroinvertebrados en el período lluvioso (e.g. Jacobsen \& Encalada, 1998; Ramírez, Pringle, \& Douglas, 2006; Ríos-Touma et al., 2011; Ríos-Pulgarín et al., 2016a).

Vásquez-Velásquez (2016), encontró en cuencas de cabecera de los Andes centrales de Colombia, que la cuenca bajo cobertura de bosque natural presentó un régimen de caudales estable y de poca variación con relación a las cuencas con cobertura de plantación forestal y cobertura en pastos, revelando una mayor inestabilidad en el régimen de caudales en éstas últimas. Así mismo la cobertura boscosa ofrece mejores condiciones de regulación de los caudales, por lo que la deforestación tiende a generar caudales máximos y mínimos más extremos (Bruijnzeel, 2004; Licata, Gyenge, Fernández, Schlichter, \& Bond, 2008; Vanclay, 2009; Vásquez-Velásquez, 2016), como se observó en la quebrada Q2-CAG, que fue la única con diferencias significativas de caudal entre los períodos seco y lluvioso.

El orden Trichoptera, y la familia Hydropsychidae, presentaron la mayor abundancia relativa para el total de individuos recolectados en las tres quebradas. Este resultado es similar a lo reportado por Jaimes-Contreras y Granados-Martínez (2016), en siete afluentes que drenan de la Sierra Nevada de Santa Marta (Colombia), y coincide también con la mayor abundancia del orden Trichoptera y la familia Hidropsychidae en el Bosque Protector Río 
Guajalito en Ecuador (Arroyo \& Encalada, 2009). La mayor abundancia de Hydropsychidae se puede relacionar con que es una familia muy tolerante, con especies segregadas dentro de diferentes características de calidad del agua a lo largo de los ríos (Bonada, ZamoraMuñoz, Rieradevall, \& Prat, 2004), y ha sido reportada como la familia más abundante en las regiones tropicales (Flint, Holzenthal, \& Harris, 1999). El amplio rango de distribución de la familia Hidropsychidae, está asociado a su alta tolerancia a cambios ambientales y su capacidad para construir redes que le permiten fijarse en el sustrato y capturar gran cantidad de alimento (López-Delgado, Vásquez-Ramos, \& Reinoso-Flórez, 2015).

Se observó una disminución del orden Tricoptera en la quebrada de cobertura de pastos (Q2-CAG), lo que coincide con lo encontrado por Henriques-Oliveira, Nessimian, y Baptista, (2015), quienes observaron una pérdida de diversidad en las quebradas sin vegetación arbórea ribereña en comparación con las quebradas con cobertura de bosque. El orden Trichoptera en sistemas lóticos se ha asociado con una alta cobertura vegetal en sus márgenes, y se ha establecido que el aumento de zonas deforestadas por actividades agropecuarias puede causar cambios ambientales y reducir su diversidad (Benstead, Douglas, \& Pringle, 2003; Lorion \& Kennedy, 2009; Breda et al., 2018). Esta menor abundancia y diversidad de Trichoptera en sitios deteriorados está relacionada con la pérdida de hábitats por procesos como la deforestación y el cambio de usos de suelo para actividades productivas, con el consecuente aumento de la sedimentación que disminuye la heterogeneidad del hábitat (Allan, Erickson, \& Fay, 1997) y la reducción de la entrada de material alóctono al cuerpo hídrico (e.g. Townsend, Dolédec, \& Scarsbrook, 1997; Bispo \& Oliveira, 2007; Bücker et al., 2010; Giraldo et al., 2014).

Para la quebrada Q2-CAG se observó una menor abundancia del orden Coleoptera y un incremento de los órdenes Diptera (e.g. Chironomidae y Simulidae) y Ephemeroptera (e.g. Leptophlebiidae y Baetidae), mientras que la quebrada Q1-CBN presentó mayores abundancias de Coleoptera (e.g. Elmidae y Ptilodactylidae). Este patrón coincide con lo encontrado por Galeano-Rendón y ManceraRodríguez (2018) quienes compararon quebradas con buena cobertura de bosque natural con quebradas sometidas a diferentes afectaciones en su cobertura por procesos de deforestación asociada con actividades agrícolas y ganaderas. Igualmente, una mayor abundancia de Ephemeroptera ha sido reportada en quebradas ubicadas en áreas deforestadas y dominadas por matrices de pastizales por Bücker et al. (2010) en el sur de Ecuador, y en Costa Rica por Lorion y Kennedy (2009), y una mayor abundancia de Díptera fue encontrada por Giraldo et al. (2014) en estaciones con intervención antrópica por ganadería en quebradas en Colombia. La mayor abundancia de Coleoptera, en particular de las familias Elmidae y Ptilodactylidae en la quebrada con cobertura de bosque natural, puede estar asociada a la mayor disponibilidad de materia orgánica gruesa y a la mayor entrada de material alóctono, ya que estas familias se han asociado con la abundancia de detritus de la hojarasca (Bojsen \& Jacobsen, 2003).

La quebrada con cobertura de bosque nativo mostró una mayor calidad hídrica y diversidad de macroinvertebrados sensibles a las perturbaciones antrópicas que las quebradas con coberturas de pastos y de plantaciones forestales. Los cambios en la composición y estructura del ensamblaje de macroinvertebrados en estas tres quebradas con diferentes coberturas riparias indican que la pérdida de vegetación nativa representa un importante impacto antrópico con efectos en la estructura de las comunidades acuáticas. En el bosque protector Murocomba la pérdida de la vegetación nativa, las malas prácticas agrícolas y el aumento de la población han modificado los usos de suelo, alterando la cobertura del suelo en los márgenes de las quebradas, la calidad hídrica y la estructura de los ensamblajes de insectos acuáticos. Por lo anterior, se recomienda el mantenimiento y restauración de la vegetación ribereña con bosques nativos, para mitigar los efectos de la 
deforestación en los patrones y procesos de las comunidades acuáticas.

Declaración de ética: los autores declaran que todos están de acuerdo con esta publicación y que han hecho aportes que justifican su autoría; que no hay conflicto de interés de ningún tipo; y que han cumplido con todos los requisitos y procedimientos éticos y legales pertinentes. Todas las fuentes de financiamiento se detallan plena y claramente en la sección de agradecimientos. El respectivo documento legal firmado se encuentra en los archivos de la revista.

\section{AGRADECIMIENTOS}

Agradecemos a la Universidad Técnica Estatal de Quevedo (UTEQ) por su apoyo logístico. Al laboratorio de Biotecnología de la Universidad Técnica de Quevedo lugar donde se identificaron los macroinvertebrados acuáticos recolectados.

\section{RESUMEN}

El cambio de uso de la tierra con áreas de bosques nativos convertidas a la agricultura, la ganadería y las plantaciones de bosques exóticos, es el factor principal que afecta la vegetación ribereña y las comunidades acuáticas. El objetivo del estudio fue evaluar las respuestas del ensamblaje de macroinvertebrados acuáticos al cambio en la cobertura ribereña en el bosque protector Murocomba, Valencia, Ecuador. Se realizaron muestreos en las temporadas seca (de junio a diciembre 2014) y de lluvias (diciembre hasta abril 2015) en tres quebradas con diferentes coberturas riparias: bosques nativos Q1-CBN, pastos para agricultura y ganadería Q2-CAG y plantaciones forestales Q3-CPF, en las que se recolectaron muestras de macroinvertebrados acuáticos y se midieron diferentes variables fisicoquímicas in situ. Según el IBF y el BMWP/Col, la mejor condición ecológica se presentó en la quebrada Q1-CBN, y el EPT disminuyó en la quebrada Q2-CAG en la estación lluviosa. El índice de ShannonWeaver mostró valores más altos en Q1-CBN y Q3-CPF en el período seco, y la dominancia aumentó en Q3-CPF en la temporada de lluvias. Un total de 6583 macroinvertebrados fueron recolectados. El orden Coleoptera mostró una mayor abundancia en Q1-CBN y Diptera en Q2-CAG, mientras que Hemiptera mostró la menor abundancia en Q3-CPF. Los taxones Psephenidae, Chironomidae, Leptophlebiidae, Veliidae, Simuliidae, Baetidae y Leptohyphidae se asociaron con los valores más altos de sólidos disueltos y $\mathrm{pH}$, así como valores más altos de IBF y valores más bajos de los índices EPT, BMWP y profundidad del canal en Q2-CAG, mientras que Leptoceridae, Elmidae, Ptilodactylidae y Philopotamidae, se asociaron con valores más altos de EPT y BMWP y con mayor profundidad en Q1-CBN y Q3-CPF. Se evidenció una influencia negativa de las actividades antrópicas en las variables fisicoquímicas y la estructura del ensamblaje de macroinvertebrados. Esto indica que la vegetación ribereña es un factor determinante para el establecimiento y la estructura de las comunidades acuáticas.

Palabras clave: insectos acuáticos; biomonitoreo; usos de suelo; conservación; Índices biológicos.

\section{REFERENCIAS}

Álvarez-Mieles, G., Irvine, K., Griensven, A. V., AriasHidalgo, M., Torres, A., \& Mynett, A. E. (2013). Relationships between aquatic biotic communities and water quality in a tropical river-wetland system (Ecuador). Environmental Science and Policy, 34, $115-127$.

Allan, D. J., Erickson, D. L., \& Fay, J. (1997). The influence of catchment land use on stream integrity across multiple spatial scales. Freshwater Biology, 37, 149-161.

Arroyo, C., \& Encalada, A. (2009). Evaluación de la calidad de agua a través de macroinvertebrados bentónicos e índices biológicos en ríos tropicales en bosque de neblina montano. Avances de Ciencia e Ingeniería, 1, 11-16.

Barba, R., Lanza, G. D. C., Ramos, A., \& González, I. (2013). Insectos acuáticos indicadores de calidad del agua en México: casos de estudio, ríos Copalita, Zimatán y Coyula, Oaxaca. Revista Mexicana de Biodiversidad, 84(1), 381-383.

Benstead, J., Douglas, M., \& Pringle, C. (2003). Relationships of Stream Invertebrate Communities to Deforestation in Eastern Madagascar. Ecological Applications, 13, 1473-1490.

Bispo, P. C., Oliveira, A. L. G., Bini, L. M., \& Sousa, K. G. (2006). Ephemeroptera, Plecoptera and Trichoptera assemblages from riffles in mountain streams of central Brazil: Environmental factors influencing the distribution and abundance of immatures. Brazilian Journal of Biology, 66(2B), 611-622.

Bispo, P. C., \& Oliveira, L. G. (2007). Diversity and structure of Ephemeroptera, Plecoptera and Trichoptera (Insecta) assemblages from riffles in mountain streams of Central Brazil. Revista Brasileira de Zoologia, 24, 283-293. 
Bojsen, B. H., \& Jacobsen, D. (2003). Effects of deforestation on macroinvertebrate diversity and assemblage structure in Ecuadorian Amazon streams. Archiv für Hydrobiologie, 158, 317-342.

Bonada, N. V., Zamora-Munoz, C., Rieradevall, M., \& Prat, N. (2004). Ecological profiles of caddis fly larvae in Mediterranean streams: implications for bioassessment methods. Environmental Pollution, 132(3), 509-521.

Breda, M., Lazari, P. L., de Oliveira, M. B., Menegat, M. N., Bertol, E. C., da Silva, G. S... Hepp, L. U. (2018). Composição e distribuição de Trichoptera (Insecta) em riachos subtropicais. Perspectiva, 42(157), 17-26.

Bruijnzeel, L. A., (2004). Hydrological functions of tropical forests: Not seeing the soil for the trees? Agriculture Ecosystems \& Environment, 104, 185-228.

Bücker, A., Sondermann, M., Frede, H. G., \& Breuer, L. (2010). The influence of land-use on macroinvertebrate communities in montane tropical streams a case study from Ecuador. Fundamental and Applied Limnology, 177, 267-282.

Carrera, P., \& Gunkel, G. (2003). Ecology of a high Andean stream, Rio Itambi, Otavalo, Ecuador. Limnologica, 33(1), 29-43.

Damanik-Ambarita, M. N., Lock, K., Boets, P., Everaert, G., Nguyen, T. H. T., Forio, M. A. E., ... Goethals, P. L. M. (2016). Ecological water quality analysis of the Guayas river basin (Ecuador) based on macroinvertebrates indices. Limnologica, 57, 27-59.

Death, R. G., \& Collier, K. J. (2010). Measuring stream macroinvertebrate responses to gradients of vegetation cover: when is enough enough? Freshwater Biology, 55, 1447-1464.

Domínguez, E., \& Fernández, H. R. (2009). Macroinvertebrados bentónicos sudamericanos. Sistemática y Biología. Tucumán, Argentina: Fundación Miguel Lillo.

Ferrú, M., \& Fierro, P. (2015). Estructura de macroinvertebrados acuáticos y grupos funcionales tróficos en la cuenca del río Lluta, desierto de Atacama, Arica y Parinacota, Chile. Idesia, 33, 47-54.

Fierro, P., Bertrán, C., Tapia, J., Hauenstein, E., PeñaCortés, F., Vergara, C., ... Vargas-Chacoff, L. (2017). Effects of local land-use on riparian vegetation, water quality, and the functional organization of macroinvertebrate assemblages. Science of the Total Environment, 609, 724-734.

Figueroa, R., Palma, A., Ruiz, V., \& Niell, X. (2007). Análisis comparativo de índices bióticos utilizados en la evaluación de la calidad de las aguas en un río mediterráneo de Chile: Río Chillán, VIII Región. Revista Chilena de Historia Natural, 80(2), 225-242.
Flecker, A. S., \& Feifarek, B. (1994). Disturbance and the temporal variability of invertebrate assemblages in two Andean streams. Freshwater Biology, 31, 131-142.

Flint, O. S., Holzenthal, R. W., \& Harris, S.C. (1999). Catalog of the Neotropical Caddisflies (Insecta: Trichoptera). Ohio, USA: Ohio Biological Survey.

Gauch, H. G. (1982). Multivariate analysis in community ecology. Cambridge, England: Cambridge University Press.

Galeano-Rendón, E., Monsalve-Cortes, L., \& ManceraRodríguez, N. J. (2017). Evaluación de la calidad ecológica de quebradas Andinas en la cuenca del Rio Magdalena, Colombia. Revista U.D.C.A Actualidad \& Divulgación Científica, 20(2), 413-424.

Galeano-Rendón, E., \& Mancera-Rodríguez, N. J. (2018). Efectos de la deforestación sobre la diversidad y la estructura del ensamblaje de macroinvertebrados en cuatro quebradas Andinas en Colombia. Revista de Biología Tropical, 66(4), DOI: 10.15517/rbt. v66i4.31397

Giacometti, J. C., \& Bersosa, F. (2006). Macroinvertebrados acuáticos y su importancia como bioindicadores de calidad del agua en el río Alambi. Boletín Técnico 6, Serie Zoológica, 2, 17-32.

Giraldo, L., Chará, J., Zúñiga, M., Chará-Serna, A., \& Pedraza, G. (2014). Impacto del uso del suelo agropecuario sobre macroinvertebrados acuáticos en pequeñas quebradas de la cuenca del río La Vieja (Valle del Cauca, Colombia). Revista de Biología Tropical, 62(Suppl. 2), 203-219.

Guerrero-Chuez, N. M., Díaz Ponce, M. A., Urdanigo Zambrano, J. P., Tayhing Cajas, C. C., Guerrero Chuez, R. V., \& Yepez Rosado, A. J. (2016). Uso de suelo y su influencia en la calidad del agua de la microcuenca El Sapanal, Ecuador. Revista Cubana de Ciencias Biológicas, 5(2), 1-11.

Guisande, C. (2015). StatR. Retrieved from http://www. ipez.es/RWizard

Hammer, Ø., Harper, D. A. T., \& Ryan, P. D. (2001). PAST: Paleontological Statistics Software Package for Education and Data Analysis. Palaeontología Electrónica. Retrieved from http://palaeoelectronica. org/2001_1/past/issue1_01.html

Henriques-Oliveira, A. L., Nessimian, J. L., \& Baptista, D. F. (2015). Diversity and composition of Trichoptera (Insecta) larvae assemblages in streams with different environmental conditions at Serra da Bocaina, Southeastern Brazil. Acta Limnologica Brasiliensia, 27(4), 394-410.

Holdridge, L. R. (1982). Ecología basada en Zonas de Vida. San José, Costa Rica: Instituto Interamericano de Cooperación para la Agricultura (IICA). 
Iñiguez-Armijos, C., Leiva, A., Frede, H. G., Hampel, H., $\&$ Breuer, L. (2014) Deforestation and benthic indicators: how much vegetation cover is needed to sustain healthy Andean streams? PLoS One, 9, e105869.

Iñiguez-Armijos, C., Rausche, S., Cueva, A., SánchezRodríguez, A., Espinosa, C., \& Breuer, L. (2016). Shifts in leaf litter breakdown along a forest-pastureurban gradient in Andean streams. Ecology and Evolution, 6, 4849-4865.

Iñiguez-Armijos, C., Hampel, H., \& Breuer, L. (2018) Land-use effects on structural and functional composition of benthic and leaf-associated macroinvertebrates in four Andean streams. Aquatic Ecology, 52(1), 77-92.

Jaimes-Contreras, A. M., \& Granados-Martínez, C. (2016) Tricópteros asociados a siete afluentes de la Sierra Nevada de Santa Marta, Colombia. Revista Mexicana de Biodiversidad, 87, 436-442.

Jacobsen, D., \& Encalada, A. (1998). The Macroinvertebrate fauna of Ecuadorian high-land streams in the wet and dry season. Archiv fur Hydrobiologie, 142(1), 53-70.

Licata, J., Gyenge, J., Fernández, M., Schlichter, T., \& Bond, B. (2008). Increased water use by ponderosa pine plantations in northwestern Patagonia, Argentina compared with native forest vegetation. Forest Ecology and Management, 255(3), 753-764.

Liñero, I., Balarezzo, V., Eraso, H., Pacheco, F., Ramos, C., Muzo, R., \& Calva, C. (2016). Calidad del agua de un río andino ecuatoriano a través del uso de macroinvertebrados. Cuadernos de Investigación UNED, 8(1), 69-75.

López-Delgado, E. O., Vásquez-Ramos, J. M., \& ReinosoFlórez, G. (2015). Listado taxonómico y distribución de los tricópteros inmaduros del departamento del Tolima. Revista Academia Colombiana de Ciencias Exactas Físicas y Naturales, 39(150), 42-49.

Lorion, C. M., \& Kennedy, B. P. (2009). Riparian forest buffers mitigate the effects of deforestation on fish assemblages in tropical headwater streams. Ecological Applications, 19(2), 468-479.

Meza, M., Rubio, J., Días, A., \& Walteros, L. (2012). Calidad de agua y composición de macroinvertebrados acuáticos en la subcuenca alta del río Chinchiná. Caldasia, 34(2), 443-456.

R Core Team. (2012). R: A language and environment for statistical computing. R Foundation for Statistical Computing. Vienna, Austria. Retrieved from https:// www.R-project.org
Ramírez, A., Pringle, C. M., \& Douglas, M. (2006). Temporal and spatial patterns in stream physicochemistry and insect assemblages in tropical lowland streams. The North American Benthological Society, 25(1), 108-125.

Ríos-Pulgarín, M. I., Barletta, M., Arango-Jaramillo, M. C., \& Mancera-Rodríguez, N. J. (2016a). The role of hydrological cycle on the patterns of macroinvertebrate assemblages in an Andean piedmont stream in Colombia. Journal of Limnology, 75(1), 107-120.

Ríos-Pulgarín, M. I., Barletta, M., \& Mancera-Rodríguez, N. J. (2016b). The role of the hydrological cycle on the distribution patterns of fish assemblages in an Andean stream. Journal of Fish Biology, 89(1), 102-130.

Ríos-Touma, B., Encalada, A., \& Prat, N. (2009). Leaf litter dynamics and its use by invertebrates in a highaltitud tropical Andean stream. International Review of Hydrobiology, 94(4), 357-371.

Ríos-Touma, B., Encalada, A., \& Prat, N. (2011). Macroinvertebrate assemblages of an Andean high altitude tropical stream: the importance of season and flow. International Review of Hydrobiology, 96(6), 667-685.

Roldán, G. (2003). Bioindicación de la calidad del agua en Colombia. Propuesta para el uso del método BMWP. Medellín, Colombia: Universidad de Antioquia.

Rosenberg, D. M., \& Resh, V. H. (1993). Freshwater biomonitoring and benthic macroinvertebrates. New York, USA: Springer.

Scarsbrook, M. R., \& Townsend, C. R., (1993). Stream community structure in relation to spatial and temporal variation: a habitat templet study of two contrasting New Zealand streams. Freshwater Biology, 29, 395-410.

Schmitt, R., Siegloch, A. E., da Silva, A. L. L., Lisboa, L. K., Petrucio, M. M. (2016). Temporal variation in the Ephemeroptera, Plecoptera and Trichoptera community in response to environmental drivers in a subtropical stream. Journal of Insect Biodiversity, 4(19), 1-12.

Terneus, E., Hernández, K., \& Racines, M. J. (2012). Evaluación ecológica del río Lliquino a través de macroinvertebrados acuáticos, Pastaza-Ecuador. Revista de Ciencias, 16, 31-45.

Townsend, C. R., Dolédec, S., \& Scarsbrook, M. (1997). Species traits in relation to temporal and spatial heterogeneity in streams: a test of habitat templet theory. Freshwater Biology, 37, 367-387. 
Vanclay, J. (2009). Managing water use from forest plantations. Forest Ecology and Management, 257(2), 385-389.

Vásquez-Velásquez, G. L. (2016). Influencia del uso de la tierra en la respuesta hidrológica de cuencas de cabecera en los Andes centrales de Colombia.(Tesis Doctoral). Universidad Nacional de Colombia.
Villamarín, C., Rieradevall, M., Paul, M. J., Barbour, M. T., \& Prat, N. (2013). A tool to assess the ecological condition of tropical high Andean streams in Ecuador and Peru: The IMEERA index. Ecological Indicators, 29, 79-92.

Zar, J. H. (1999). Biostatistical Analysis. New Jersey, USA: Prentice-Hall. 